\title{
IDEAS PREVIAS EN LA BOTÁNICA
}

\author{
MATEOS JIMENEZ, A. \\ Departamento de Didáctica de las Ciencias Experimentales. Universidad de Castilla - La Mancha. \\ Escuela Universitaria de Formación del Profesorado de EGB. Av, de Barber s/n. 45005 Toledo.
}

\section{SUMMARY}

We have found the same kind of botanical preconcepts among students of 8th EGB and students of the Teacher-training School from Toledo. If students have studied sciences the previous years these preconcepts seem less abundant. The most widespread is that pine-cone is the fruit of pines trees. Other beliefs are referred to flowers, trees, potatoes, fruits, and seeds.

\section{INTRODUCCIÓN}

Existe un creciente interés dentro del campo de la enseñanza de las ciencias por conocer las ideas previas de los alumnos para, a partir de ellas, diseñar estrategias docentes acordes que permitan un aprendizaje significativo. Esta tendencia se cimenta en el constructivismo y en las aportaciones de Ausubel et ai. (1983).

Las ideas previas son conocidas en la bibliografía con diversas denominaciones: desde «ideas acientificas» a «concepciones alternativas» (Driver et al., Jiménez Aleixandre 1989). Sus características han sido bien descritas por varios autores (Driver 1986, Driver et al. 1989, Harlen 1989) y algunos de sus orígenes han sido, recientemente, revisados por Pozo y otros (1991). En ocasiones parecen responder a una necesidad de los alumnos de «organizar» sus experiencias cotidianas (Harlen 1989) aunque, en algunos conocimientos biológicos, se ha propuesto una influencia notable de factores culturales (Deadman y Kelly 1978, Hewson 1985).

Dentro de este enfoque constructivista se enmarcan las aportaciones referidas al aprendizaje como cambio conceptual (Posner et al. 1982) o al modelo generativo del aprendizaje (Osborne y Wittrock 1983). A su vez, se insiste en que el cambio conceptual debe propiciarse a partir de un cambio metodológico (Carrascosa y Gil 1985, Gil 1986, Gené 1991, Jiménez Aleixanđie 1991, Segura 1991).

Son numerosos los estudios sobre preconcepciones en física y química, tal y como se puede comprobar en las recientes revisiones de Carmichael y otros (1990) y Pessoa de Carvalho y otros (1991). En biología son más escasos (Serrano 1987, Carmichael et al. 1990). Aun así, su mención sería en exceso prolija dado que abordan numerosos ámbitos biológicos, a saber: biología celular (Dreyfus y Jungwirth 1988), fisiología vegetal (CañaI y García 1987, Giordan y De Vecchi 1988, Wood-Robinson 1991), fisiología animal (Serrano 1988, Banet y Núñez 1992, Carrasquer y Álvarez Sevilla, en prensa), reproducción (Carmichael et al. 1990, Fontes y Duarte 1992), ecología (García et al. 1990), evolución (Lucas 1971 , Jiménez Aleixandre 1989, Gené 1991) y sistemática zoológica (Bell 1981, Trowbridge y Mintzes 1988, Velasco 1991).

Dentro de la botánica, Ias ideas previas relativas a la organografía constituyen una línea todavía poco abonada aunque pueden citarse irabajos centrados en casi todas las estructuras vegetales tanto vegetativas como reproductoras (Symington et al. 1981, Buiza et al. 1983, Buiza et al. 1988, Diaz de Bustamante et al. 1989. Jiménez Aleixandre 1989, Wood-Robinson 1991, Guzmán 1992).

A la vista de la importancia actual de las ideas alternativas dentro del proceso de enseñanza-aprendizaje y teniendo en cuenta la trascendental labor futura de los alumnos empleados como muestra (futuros maestros), el presente estudio se plantea, como objetivo, responder a los siguientes interrogantes:

- ¿Coinciden y, por lo tanto, persisten, las ideas previas en la botánica en alumnos de distinto nivel educativo?

- ¿Cuáles son, en cada caso, estas ideas previas?

- ¿Existen diferencias entre Ias ideas previas de alumnos del mismo nivel educativo pero con distinta preparación científica? 


\section{METODOLOGÍA}

Muestra: 216 alumnos de la ciudad de Toledo, 172 de ellos de la Escueia Universitaria de Formación del Profesorado de EGB y 44 de $8^{\circ}$ curso de EGB (próxima Educación Primaria), por lo tanto, de niveles educativos distintos y bien diferenciados.

Los alumnos de Magisterio pertenecían a $1^{\circ}, 2^{2}$ y 3 er curso de especialidades «no científicas» (Humanas, Filología y Preescolar) y a $1^{\circ}$ y $2^{\circ}$ de la especialidad de Ciencias. Puede entenderse, en general, que el grupo «no ciencias» (NCC) posee, en principio, menor preparación científica que el de ciencias (CC) al provenir, en su mayoría, de un $3^{\circ}$ de BUP y COU de letras o mixto.

Aproximadamente la mitad de los futuros maestros procedían de ambientes rurales.

\section{Instrumento}

Se aplicó un cuestionario a todos los alumnos de la muestra. Dicho cuestionario fue elaborado por el propio autor del trabajo teniendo en cuenta la experiencia docente acumulada como profesor de escuela de magisterio y las opiniones y propuestas concretas de profesores de la todavía vigente $2^{a}$ etapa de EGB, en el ámbito de las ciencias naturales.

El cuestionario se aplicó, en todos los casos, antes de abordar los puntos de botánica en sus respectivas disciplinas científicas. Lo componían ítems de elección múltiple cada uno con 4 posibles respuestas de las cuales una siempre permitía la posibilidad de contestar «no sé» (véase Anexo). Se debía escoger una opción y marcarla con X.

\section{ANÁLISIS DEL CUESTIONARIO}

EI cuestionario albergaba conceptos relativos al fruto, semilla, flores e inflorescencias. Asimismo planteaba la definición y naturaleza botánica de especies de uso doméstico como la zanahoria (Daucus carota) y la patata (Solanum tuberosum).

Con las preguntas se pretendió comprobar, en concreto, si los alumnos identificaban la piña como el fruto de los pinos o como la inflorescencia femenina transformada (Font Quer 1979), si poseían un correcto conocimiento de la semilla y del embrión y si relacionaban las flores ślo con los pétalos o también con el androceo y gineceo. Asimismo, podríamos comprobar si establecían diferencias entre los conceptos de fruta y fruto y, por último, si eran capaces de saber qué estructura floral es una marga* rita (Gén. Leucanthemun).

Todos estos contenidos, tal vez a excepción de la inflorescencia en el capítulo de la margarita, suelen incluirse tanto en EGB como en el bachillerato y COU.
Las cuestiones se procuraron construir con un lenguaje asequible y directo. En la número 3 se introdujo, por tal razón, el término «echar flores» en vez de «tener» flores.

\section{RESULTADOS}

La tabla I muestra que la gran mayoría de los alumnos admiten la existencia de fruto y semilla en los pinos y definen la piña como el fruto del pino. Esta última idea está tan extendida entre los alumnos de las especialidades «no científicas» (64\%) como en los de ciencias (64\%). Esta creencia es todavía mayor entre los escolares ( $73 \%$ ).

Por el contrario, sí reconocen, en su mayoría, el piñón como semilla ( $68 \%$ en CC, $55 \%$ en NCC, y $57 \%$ en $8^{\circ}$ de EGB). A su vez, el porcentaje de alumnos que lo confunden con el fruto es, aproximadamente, el doble en «no ciencias» $(31 \%)$ que en ciencias $(16 \%)$.

Los alumnos, en general, dudan de que todos los árboles posean flores (el $65 \%$ en CC, el $76 \%$ en NCC y el $93 \%$ en EGB, sumando las respuestas 3.2 «ninguno» y 3.3 "sólo algunos»). Asimismo, la mayoría creen que una flor debe tener siempre pétalos $(51 \%$ en $\mathrm{CC}, 68 \%$ en NCC y $73 \%$ en EGB, sumando las respuestas 4.1 y 4.3 ).

Parece conocido por todos los alumnos $(74,19 \%$ en $\mathrm{CC}$, $73,04 \%$ en $\mathrm{NCC}$ y $61,36 \%$ en $\mathrm{EGB}$ ) que la zanahoria representa una ráiz modificada y engrosada. Erróneamente, la mayoría también define la patata como una raíz subterránea engrosada $(64,51 \%$ en $\mathrm{CC}, 58,68 \%$ NCC y $56,81 \%$ en EGB).

Para la mayoría de alumnos de «no ciencias» $(51,77 \%)$ y $\operatorname{EGB}(61,36 \%)$, fruto y fruta es distinta cosa. En cambio, la mayoría de los alumnos de la especialidad de ciencias creen que es lo mismo $(58,06 \%)$.

En una planta como la margarita, de fuerte arraigo popular, se desconoce abrumadoramente $(67,74 \%$ en CC, $70,92 \%$ en NCC y $65,92 \%$ en EGB) que es una inflorescencia en capítulo o cabezuela, formada por dos tipos de flores.

A la hora de localizar el embrión en la semilla, alrededor de la mitad de los alumnos, aproximadamente, lo ubican dentro de la semilla $(51,06 \%$ en CC y $45,39 \%$ en NCC). Curiosamente, en EGB este porcentaje es algo mayor $(68,18 \%)$.

Finalmente, algo menos del $50 \%$, aproximadamente, de todos los alumnos creen que una semilla es tanto el hueso de una aceituna como un guisante.

\section{CONCLUSIONES}

El tamaño de la muestra nos invita a ser cautos a la hora de extraer conclusiones. No obstante, pueden apuntarse las siguientes: 
Tabla I

Porcentajes aproximados de cada respuesta.

* Respuesta correcta.

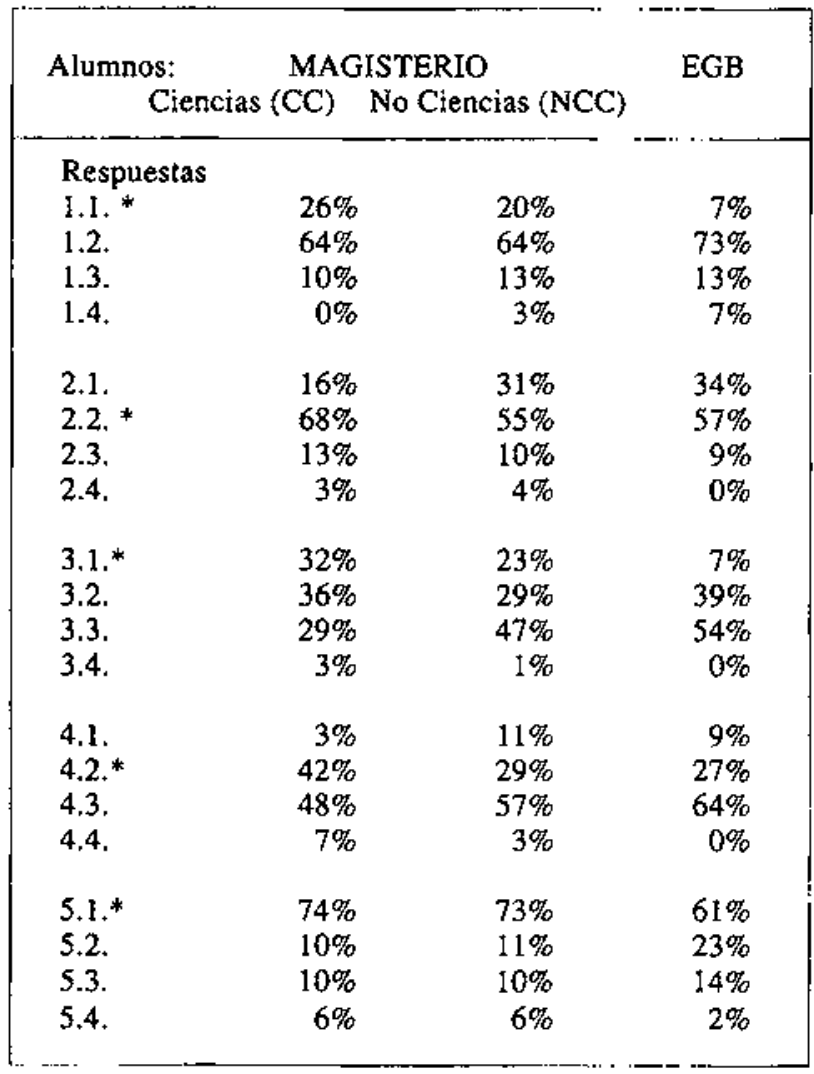

La idea previa más extendida entre los alumnos de Magisterio y EGB es que la piña es el fruto del pino. Se puede considerar que esta concepción es errónea desde el punto de vista organogénico como indica Font Quer (1979) y también desde el punto de vista de la «ciencia escolar» (según la denominación de Osborne y Freyberg 1990), en donde el fruto es el ovario maduro. No obstante, como también señala Font Quer (1979), si se adopta un criterio ecológico en vez de organogénico, existe una tendencia que considera fruto a lo que encierra a las semillas.

Desconocemos si la idea piña $\approx$ fruto puede tener igual justificación en Magisterio que en EGB. No parece prudente adelantar una posible explicación. Entre otras consideraciones, tal vez, pudiera estar arraigada la creencia de la «obligatoriedad» del fruto y la semilla para todas las plantas o también podría intervenir laidea de continente (fruto) que alberga un contenido «duro» («hueso» = semilla), o incluso pudiera partir únicamente de un escaso conocimiento teórico-práctico de la estructura reproductora femenina de las gimnospermas. Este desconocimiento ha sido también apuntado por Díaz de Bustamante y otros (1989) con alumnos de $8^{\circ}$ de EGB y Magisterio, en relación con distintas especies de árboles, y por Guzmán (1992) con alumnos de Magisterio, para las angiospermas.
Tabla II

Porcentajes aproximados de cada respuesta.

* Respuesta correcta.

\begin{tabular}{|c|c|c|c|}
\hline \multicolumn{3}{|c|}{$\begin{array}{c}\text { Alumnos: } \\
\text { Ciencias (CC) No Ciencias (NCC) }\end{array}$} & EGB \\
\hline \multicolumn{4}{|c|}{ Respuestas } \\
\hline 6.1 & $65 \%$ & $59 \%$ & $57 \%$ \\
\hline 6.2. * & $19 \%$ & $18 \%$ & $16 \%$ \\
\hline 6.3 . & $13 \%$ & $21 \%$ & $25 \%$ \\
\hline 6.4 & $3 \%$ & $2 \%$ & $2 \%$ \\
\hline 7.1. * & $58 \%$ & $44 \%$ & $18 \%$ \\
\hline 7.2 & $32 \%$ & $34 \%$ & $61 \%$ \\
\hline 7.3. & $10 \%$ & $14 \%$ & $16 \%$ \\
\hline 7.4 . & $0 \%$ & $8 \%$ & $5 \%$ \\
\hline 8.1 . & $68 \%$ & $71 \%$ & $66 \%$ \\
\hline 8.2 & $13 \%$ & $9 \%$ & $11 \%$ \\
\hline 8.3.* & $16 \%$ & $8 \%$ & $9 \%$ \\
\hline 8.4 & $3 \%$ & $12 \%$ & $14 \%$ \\
\hline 9.1. & $32 \%$ & $41 \%$ & $23 \%$ \\
\hline 9.2.* & $52 \%$ & $49 \%$ & $68 \%$ \\
\hline 9.3 & $10 \%$ & $1 \%$ & $2 \%$ \\
\hline 9.4 & $6 \%$ & $9 \%$ & $7 \%$ \\
\hline $10.1 .^{*}$ & $48 \%$ & $39 \%$ & $61 \%$ \\
\hline 10.2 & $32 \%$ & $16 \%$ & $14 \%$ \\
\hline 10.3 & $16 \%$ & $29 \%$ & $18 \%$ \\
\hline 10.4. & $4 \%$ & $16 \%$ & $7 \%$ \\
\hline
\end{tabular}

Podría considerarse que la idea del «continente-contenido» es capaz de explicar, junto a otras, por qué identifican los piñones con la semilla, ya que, según esta visión, estarían dentro del supuesto fruto (piña). No obstante, creemos que este esquema, aunque es coincidente con el criterio ecológico apuntado por Font Quer (1979), difícilmente responde al mismo planteamiento previo $\mathrm{nj}$ tiene la misma argumentación. Esta relación piña = fruto puede hallarse, a su vez, en libros de texto de EGB afianzando esta idea previa escolar. En Magisterio, dada la futura labor de los alumnos, la persistencia de dicha idea parece más preocupante.

La identificación «continente-contenido» tal vez también pueda contribuir a que los alumnos equiparen huesos con guisantes.

La ausencia de flores en algunos árboles es una idea previa que se mantiene en nuestra muestra, habiéndose citado también en otros trabajos (Buiza et al. 1988, Díaz de Bustamante et al. 1989, Jiménez Aleixandre 1989).

Podemos pensar que una parte del alumnado otorga todavía a los conceptos de fruto y fruta distinto signifícado botánico, tal vez condicionados por el lenguaje doméstico y tradicional procedente de su utilidad gastronómica (Font Quer 1979) o por otros motivos diferentes. 
Esta diferenciación tal vez no influya decisivamente en la adquisición de futuros conceptos botánicos pero resulta algo parad6jica si quienes la presentan son futuros maestros. Buiza y otros (1988) detectan una identificación del fruto sólo con la fruta en alumnos de $1^{\circledR}$ de BUP.

Finalmente, constatamos que es mayoritariamente desconocido que la margarita es una inflorescencia. Ello podría deberse a diversos motivos entre los cuales no se puede descartar la tradición sociocultural que identifica las flores ligulares con los pétalos de Ia supuesta única flor, o incluso por el pequeño tamaño de la flores tubulares y la disposición oculta de parte de la flor ligular. Esto abundaría en el origen histórico-cultural de algunas preconcepciones (Pozo et al. 1991). Jiménez Aleixandre (1989) alude también al efecto distorsionador que tendrían los dibujos excesivamente arquetípicos sobre flores, existentes en numerosos textos escolares.

Se ha detectado, en general, que los alumnos de la especialidad de Ciencias respondieron más acertadamente al cuestionario que los de otras especialidades. Los de $8^{\circ}$ de EGB presentaron, a menudo, porcentajes semejantes a los alumnos de NCC. No se han hallado diferencias importantes entre alumnos de zonas rurales y no rurales. Trabajos como los de Díaz de Bustamante y otros (1989) señalan leves diferencias entre alumnos de distintos cursos de EGB y Magisterio.

\section{CONSIDERACIONES FINALES}

Nuestras conclusiones apuntan a que diversos conceptos básicos de la botánica no parecen asumidos por los alumnos investigados, ya sean de EGB o de niveles universitarios iniciales. Estos conceptos son la base para la progresión en botánica. Buiza y otros (1988) y Jiménez Aleixandre (1989) relacionan este hecho con los modelos de instrucción utilizados, que se apoyan más en ejemplos ideales de flores y frutos que en ejemplos reales. No debe tampoco olvidarse la influencia que puede tener el lenguaje de los alumnos en la construcción de dichas ideas (Llorens 1989).

Estas ideas previas ya aparecen en $8^{\circ}$ de EGB y no parecen modificarse a pesar de los posteriores cursos de ciencias en $1^{2}$ y $3^{9}$ de BUP e incluso COU. Particularmente preocupante es su persistencia entre los futuros maestros.

Parece necesario un esfuerzo docente continuado que permita superar estas y otras posibles ideas previas. Para ello, deberían escogerse, como material de estudio, ejemplos muy diversos de especies botánicas evitando así la simplificación que presentan ciertos libros de texto. En tal sentido, esquemas de instrucción que emplean los casos conocidos por los alumnos junto a casos poco conocidos e incluso desconocidos para ellos (Llopis y Serrano 1981) podrían servir de base para su aplicación botánica ya desde los niveles educativos inferiores.

Debe insistirse en la importancia que tiene un adecuado apoyo audiovisual a la hora de abordar las estructuras botánicas, unido a la incuestionable utilidad de las sesiones prácticas de reconocimiento botánico. En este sentido, la elaboración (prudente) de herbarios y el estudio con el microscopio estereoscópico de los ejemplares recolectados puede propiciar la extensión de las ideas previas constituyendo así un ejemplo de «captura conceptual» (Jiménez Aleixandre 1989). Autores como Díaz de Bustamante y otros (1989) subrayan para este fin el valor del estudio de ejemplares de especies botánicas cercanas al alumno.

\section{Nota}

Este trabajo se ha financiado con una ayuda del Departamento de Pedagogía de la Universidad de Castilla - La Mancha. 


\section{REFERENCIAS BIBLIOGRÁFICAS}

AUSUBEL, D.P., NOVAK, J.D. Y HANESIAN, H., 1983. Psicología Educativa: Unpunto de vista cognoscitivo.(Trillas: Méjico),

BANET, E. y NÚŇEZ, F, 1992. La digestión de los alimentos: un plan de actuación en el aula fundamentado en una secuencia constructivista del aprendizaje, Enseñanza de las Ciencias, 10(2), pp. 139-147.

BELL, B.J., 1981. When is an animal, not an animal?, Journal of Biological Education, 15(3), pp. 213-218.

BUIZA, C., MARTÍN, N., NIEDA, J. y SENANTE, F., 1983. Los árboles. (Ayuntamiento de Madrid: Madrid).

BUIZA, C., MARTÍN, N., NIEDA, J. y SENANTE, F., 1988. Cómo corregir errores conceptuales en botánica utilizando una estrategia de trabajo. (Trabajo inédito).

CAÑAL, P. y GARCÍA, S., 1987. La nutrición vegetal, un año después. Un estudio de un caso en séptimo de EGB, Investigación en la Escuela, 3, pp. 55-60.

CARMICHAEL, P., DRIVER, R., HOLDING, B., PHILLIPS, I., IWIGGER, D. y WATIS, M., 1990. Research on children's conceptions in science: a bibliography. (University of Leeds: Leeds).

CARRASCOSA, J. y GLL, D., 1985. La metodología de la superficialidad y el aprendizaje de las ciencias, Enseñanza de las Ciencias, 3(2), pp. 113-120.

CARRASQUER, J. y ÁLVAREZ SEVILLA, M.V., 1992. Consideraciontes acerca de de la ciencia de los alumnos en el proceso de respiración extema de animales acuáticos. Actas de los XIII Encuentros de Didáctica de las Ciencias Experimentoles. Guadalajara. (En prensa).

DEADMAN, J.A. y KELLX, P., 1978. What secondary school boys understand about evolution and heredity before they are taught the topics?, Journal of Biological Education, 12 (1), pp. 7-15.

DIAZ DE BUSTAMANTE, J., GARCIA, F. y GARCIARODEJA, I., 1989. Flores, froitos, sementes... Ideas dos nenos sobre as partes das plantas, Boletin das Ciencias, Octubre, pp. 70-76.

DREYFUS, A. y JUNGWIRTH, E., 1988. The cell concept of 10th graders: curricular expectations and reality, International Journal of Science Education, 10 (2), pp. 221-229.

DRIVER, R,, 1986. Psicología cognoscitiva y esquemas conceptuales de los alumnos, Enseñanza de las Ciencias, $4(1)$, pp. 3-15.

DRIVER, R., GUESNE, E. y TIBERGHEIN, A., 1989. Ideas cientificas en la infancia y adolescencia. (Morata: Madrid).

FONTES, M.A. y DUARTE, M.C., 1992. Creencias populares y enseñanza de la Biología, Enseñanza de las Ciencias, 10(1), pp. $89-93$.

FONT QUER, P., 1979. Diccionario de Botanica. (Labor: Madrid).

GARCIA, J.E., LLEDO, A., PEDRINACI, E. y GARCÍA, S., 1990. Concepciones de los alumnos sobre el concepto de interacción biológica, VII Jornadas de Estudio sobre la Investigación en la Escuela, citado en Pozo, J.A., Sanz, A., Gómez Crespo, M.A. y Litnon, M., 1991. Las ideas de los alumnos sobre la Ciencia: una interpretación desde la psicología cognitiva, Enseñanza de las Ciencias, 9(1), pp. 83-94.

GENÉ, A., 1991. Cambio conceptual y metodología en la enseñanza y el aprendizaje de la evolución de los seres vivos. Un ejemplo concreto, Enseñanza de las Ciencias, 9(1), pp. 22-27.

GIL, D., 1986. La metodología científica y la enseñanza de las ciencias: unas relaciones controvertidas, Enseñanza de las Ciencias, 4 (2), pp. 111-121.

GIORDAN, A. y DE VECCHI, G., 1988. Los orfgenes del saber. (Diada: Sevilla).

GUZMÁN, D., 1992. Ideas previas sobre la morfología de la flor y de la hoja en alumnos de Magisterio. Actas XII Encuentro de Didáctica de las Ciencias Experimentales, pp. 437-441.

HARLEN, N., 1989. Enseñanza y aprendizaje de las Ciencias. (Morata: Madrid).

HEWSON, P., 1981. A conceptual change approach to learning science, European Journal of Science Education, 3 (4), pp. 383-396.

HEWSON, M., 1985. The role of intelectual environment in the origin of conceptions: an exploratory study, en West y Pine (eds.), Cognitive structure and conceptual change, pp. 153161 .

JIMÉNEZ ALEIXANDRE, M.P., 1989. La ciencia de los y las adolescentes: esquemas conceptuales en biologia. (ICE Universidad de Zaragoza: Zaragoza).

JIMÉNEZ ALEIXANDRE, M.P., 1991. Cambiando las ideas sobre el cambio biológico, Enseñanza de las Ciencias, 9(3), pp. 248-256.

LUCAS, A.M., 1971. The teaching of «adaptation», Journal of Biological Education, 5, pp. 86.90.

LLOPIS, C. y SERRANO, M.T., 1981. El area de experiencias en Preescolar y ciclo preparatorio. (Narcea: Madrid).

LLORENS, J.A., 1989. La función del lenguaje en un enfoque constructivista del aprendizaje, Enseñanza de las Ciencias $7(2)$, pp. 111-119.

OSBORNE, R. y FREYBERG,P.,(ed.) 1990. El aprendizaje de las Ciencias: las implicaciones de la Ciencia infantil. (Narcea: Madrid).

OSBORNE, R. y WITTROCK, M.C., 1983. Leaming Science: a generative process, Science Education, 67(4), pp. 489-508.

PESSOA DE CARVALHO, A.M.,LABURU, C.E., DA SILVA, D., MORTIMER, E., GARRIDO, E., TRIVELATO, J., BECHARA, L., ORIOSVALDO DE MOURA, M., GONÇALVES, M.E., SANTOS, M., TEIXEIRA, O.P., RIBEIRO DO VALLE, M., DE CASTRO, R. y ITACARAMBI, R., 1991. Síntesis evolutivade investigaciones en enseñanza de Ciencias, Enseñanza de las Ciencias, 9(2), pp. 169-174.

POSNER, G., STRIKE, K., HEWSON, P. y GERTZOG, W. 1982. Accomodation of a scientific conception: toward a teory of conceptual change, Science Education, 66(2), pp. 489-508. 
POZO, J.A., SANZ, A., GOMEZ CRESPO, M.A. y LIMON, M., 1991. Las ideas de los alumnos sobre la Ciencia: una interpretación desde la psicología cognitiva, Enseñanza de las Ciencias, 9 (1), pp. 83-94.

SEGURA, D., 1991. Una premisa para el cambio conceptual: el cambio metodologico, Enseñanza de las Ciencias, 9(2), pp. $175 \cdot 180$.

SERRANO, T., 1987. Representaciones de los alumnos en Biología: estado de la cuestion y problemas para su investigacion en el aula, Enseñanza de las Ciencias, 5(3), pp. 181-188.

SERRANO, T., 1988. Reconstruir las ideas de los alumnos. Representaciones sobre el sistema nervioso, Investigación en la Escuela, 6, pp. 95-107.
SYMINGTON, D., BOUNDY,K, RADFORD, T. y WALTON, J., 1981. Children's drawing of natural phenomena, Research in Science Education, 11, pp. 44-51.

TROWBRIDGHE, J.E. y MINTZES, J.J., 1988. Alternative conceptions in animal classification: across-age study, Journal of Research in Science Teaching, 25(7), pp. 547-571.

VELASCO, J.M., 1991. ¿Cuándo un servivo puede ser considerado animal?, Enseñanza de las Ciencias, 9(1), pp. 43-52.

WOOD-ROBINSON, C., 1991. Young people's ideas about plants, Studies in Science Education, 19, pp. 119-135. 


\section{ANEXO}

Encuesta botánica.

Elige una sola respuesta y márcala $(\mathrm{X})$.

1. ¿Qué es la piña de un pino?
1.1 ( ) Las flores femeninas transformadas
1.3 ( ) La semilla
$1.2($ ) E! fruto
1.4 ( ) No sé

2. ¿Qué son los piñones de un pino?
2.1 ( ) El fruto
2.3 () Nada de lo anterior
2.2 ( ) La semilla
2.4 ( ) No sé

3. ¿Crees que todos los árboles «echan» flores?
3.1 ( ) Si
3.3 ( ) S6́lo algunos árboles
3.2 ( ) Ninguno
3.4 () No sé

4. En tu opinion, una flor, para que sea flor, debe tener siempre:
4.1 ( ) Pétalos
4.3 ( ) Las 3 cosas a la vez
4.2 ( ) Estambres y/o pistilo
4.4 () No sé

5. ¿Sabrías decir qué es reaimente una zanahoria?
5.1 ( ) Una raíz subterránea engrosada
5.3 ( ) Nada de esas dos cosas
5.2 ( ) Un tallo subterráneo engrosado
5.4 ( ) No sé

6. ¿Sabrías decir qué es realmente una patata?
6.1 ( ) Una raiz subterránea engrosada
6.3 ( ) Nada de esas dos cosas
6.2 () Un tallo subterráneo engrosado
6.4 ( ) No sé

7. Los frutos (aceitunas, beilotas, etc.) y las frutas (peras, naranjas etc.), ¿son lo mismo?
7.1 ( ) Sí, pero con distinto nombre
7.3 ( ) Depende del árbol
7.2() No
7.4 () No sé

8. La margarita ¿está formada por una única flor?
8.1 ( ) Sí
8.3 ( ) No. La forman 2 tipos de flores
8.4 () No sé
9. En una semilla, ¿dónde está el embrión de la futura planta?
9.1 ( ) Es la propia semilla
9.3 () No tiene
9.2 ( ) Dentro de la semilla
9.4 ( ) No sé

8.2 ( ) No. La forman varios tipos de flores

10. El hueso de una aceituna y un guisante, ¿son los dos semillas?
10.1 ( ) Sí
10.3 () El guisante no
10.2 ( ) El hueso no
10.4 ( ) No sé 